\title{
On spectral continuity of positive elements
}

\author{
by \\ S. Mouton (Stellenbosch)
}

\begin{abstract}
Let $x$ be a positive element of an ordered Banach algebra. We prove a relationship between the spectra of $x$ and of certain positive elements $y$ for which either $x y \leq y x$ or $y x \leq x y$. Furthermore, we show that the spectral radius is continuous at $x$, considered as an element of the set of all positive elements $y \geq x$ such that either $x y \leq y x$ or $y x \leq x y$. We also show that the property $\varrho(x+y) \leq \varrho(x)+\varrho(y)$ of the spectral radius $\varrho$ can be obtained for positive elements $y$ which satisfy at least one of the above inequalities.
\end{abstract}

1. Introduction. The subject of spectral continuity has been studied for more than fifty years, and several authors have contributed; in particular, by providing different types of sufficient conditions for spectral continuity. In her survey paper [2] of 1994, L. Burlando gave an extensive account of these results, and supplied many useful references.

It is well known that if $A$ is a noncommutative Banach algebra, then the spectrum and spectral radius functions are only upper semicontinuous on $A$, while if $A$ is a commutative Banach algebra, then these functions are uniformly continuous on $A$. More generally, if $x \in A$, then $\operatorname{Sp}(y) \subset \operatorname{Sp}(x)+$ $\varrho(x-y)$ for all $y \in\{x\}^{c}$ (see [1, Theorem 3.4.1]), and hence $|\varrho(y)-\varrho(x)| \leq$ $\varrho(x-y)$ for all $y \in\{x\}^{\mathrm{c}}$ (where Sp denotes the spectrum, $\varrho$ the spectral radius and $\{x\}^{\mathrm{c}}$ the commutant $\{y \in A: y x=x y\}$ of $x$ ), so that the spectral radius is continuous at $x$, considered as an element of $\{x\}^{\mathrm{c}}$.

In this paper we investigate certain spectral continuity properties of positive elements. Some spectral theory of positive elements in ordered Banach algebras was developed in [8] and [7], and later in [4]-[6]. We recall some of this information in Section 3. In Section 4 we show that if $x$ is a positive element of an ordered Banach algebra, then the results mentioned above can be obtained under the weaker condition that either $x y \leq y x$ or $y x \leq x y$, provided that $x \leq y$ and (for some results) one of a number of additional spectral properties is assumed. In Section 5 we give examples to show that these spectral properties are quite natural.

2000 Mathematics Subject Classification: 46H05, 47A10, 47B65, 06F25.

Key words and phrases: ordered Banach algebra, positive element, spectrum. 
Another well known spectral property is that if $x$ and $y$ commute, then $\varrho(x+y) \leq \varrho(x)+\varrho(y)$ and $\varrho(x y) \leq \varrho(x) \varrho(y)$. It is already known (see [8, Proposition 4.4]) that the latter inequality can still be obtained for positive elements $x$ and $y$ if, instead of commuting, they satisfy at least one of the inequalities $x y \leq y x$ and $y x \leq x y$. The problem of finding conditions under which $\varrho(x+y) \leq \varrho(x)+\varrho(y)$ will hold, for positive elements $x$ and $y$ satisfying at least one of the inequalities $x y \leq y x$ and $y x \leq x y$, was investigated in [3] and in [10], where $x$ and $y$ were bounded linear operators on a partially ordered Banach space and on a Banach lattice, respectively. Furthermore, in [11], the same problem was studied for the local spectral radius (instead of the spectral radius) of a bounded linear operator. We show (in Section 4) that if either $x y \leq y x$ or $y x \leq x y$ with $x$ and $y$ positive, then $\varrho(x+y) \leq$ $\varrho(x)+\varrho(y)$ always holds, provided that the algebra cone is normal. The result is applicable, for instance, in the case of the bounded linear operators on the Banach lattice $l^{p}($ any $p)$-see Example 5.2.

2. Preliminaries. Throughout, $A$ will be a complex Banach algebra with unit 1. The spectrum of an element $x$ in $A$ will be denoted by $\operatorname{Sp}(x)$, the spectral radius of $x$ in $A$ by $\varrho(x)$, and the distance $d(0, \operatorname{Sp}(x))$ from 0 to the spectrum of $x$ by $\delta(x)$. We recall that if $\alpha \notin \operatorname{Sp}(x)$, then $d(\alpha, \operatorname{Sp}(x))=$ $1 / \varrho\left((\alpha 1-x)^{-1}\right)([1$, Theorem 3.3.5]). If $K$ is a compact set in $\mathbb{C}$ and $r>0$, then $K+r$ denotes the set $\{z \in \mathbb{C}: d(z, K) \leq r\}$, and $C(0, r)$ the circle in the complex plane with centre 0 and radius $r$. If $r=0$, then $C(0, r)$ denotes the one-point set $\{0\}$. Finally, we need the following lemma:

LEMMA 2.1 ([1, proof of Corollary 3.2.10]). Let $a$ and $b$ be elements of $a$ Banach algebra $A$, and let $\lambda, \mu \in \mathbb{C}$ and $n \in \mathbb{N} \cup\{0\}$. Then:

(1) $\left\|\sum_{k=0}^{n}\left(\begin{array}{l}n \\ k\end{array}\right)(\lambda a)^{n-k}(\mu b)^{k}\right\|^{1 / n} \leq(\lambda+\mu)\left(\max _{0 \leq k \leq n}\left\|a^{n-k}\right\|\left\|b^{k}\right\|\right)^{1 / n}$.

(2) If $\varrho(a)<1, \varrho(b)<1$ and $\gamma_{n}=\max \left\{\left\|a^{2^{n}-k}\right\|\left\|b^{k}\right\|: 0 \leq k \leq 2^{n}\right\}$, then there exists an $N \in \mathbb{N}$ such that $\left(\gamma_{n}\right)$ is decreasing for $n \geq N$.

3. Ordered Banach algebras. In [8, Section 3] we defined an algebra cone $C$ of a Banach algebra $A$ and showed that $C$ induced on $A$ an ordering which was compatible with the algebraic structure of $A$. Such a Banach algebra is called an ordered Banach algebra. We now recall those definitions and also the additional properties that $C$ may have.

Let $A$ be a complex Banach algebra with unit 1. Suppose that $A$ contains a subset $C$ with the following properties:

(1) $C+C \subseteq C$,

(2) $\lambda C \subseteq C$ for all $\lambda \geq 0$, 
(3) $C \cdot C \subseteq C$,

(4) $1 \in C$.

Then $C$ is called an algebra cone of $A$, and $A$, or more specifically $(A, C)$, is called an ordered Banach algebra (OBA). We say that $A$ is ordered by the algebra cone $C$. If, in addition, $C \cap-C=\{0\}$, then $C$ is called proper.

An algebra cone $C$ of $A$ induces an ordering " $\leq$ " on $A$ in the following way:

$$
x \leq y \quad \text { if and only if } \quad y-x \in C
$$

$(x, y \in A)$. This ordering is reflexive and transitive. Furthermore, $C$ is proper if and only if the ordering has the additional property of being antisymmetric. Considering the ordering that $C$ induces we find that $C=\{x \in A: x \geq 0\}$ and therefore we call the elements of $C$ positive.

An algebra cone $C$ of $A$ is called closed if it is a closed subset of $A$. Furthermore, $C$ is said to be normal if there exists a constant $\alpha>0$ such that it follows from $0 \leq x \leq y$ in $A$ that $\|x\| \leq \alpha\|y\|$. It is well known that if $C$ is normal, then $C$ is proper. Moreover, $C$ is said to be inverse-closed if it has the property that if $x \in C$ and $x$ is invertible, then $x^{-1} \in C$.

The following lemma is immediate:

Lemma 3.1. Let $(A, C)$ be an $O B A$, and let $x, y \in A$ be such that $x y \leq y x$.

(1) If $x$ is invertible with $x^{-1} \in C$, then $y x^{-1} \leq x^{-1} y$.

(2) If $y$ is invertible with $y^{-1} \in C$, then $y^{-1} x \leq x y^{-1}$.

The next result follows by induction:

Lemma 3.2. Let $(A, C)$ be an $O B A$, and let $x, y \in C$. If $y x \leq x y$, then

$$
(x+y)^{n} \leq \sum_{k=0}^{n}\left(\begin{array}{l}
n \\
k
\end{array}\right) x^{n-k} y^{k}
$$

for every $n \in \mathbb{N} \cup\{0\}$.

Proof. Clearly the statement is true for $n=0$ (and $n=1$ ). So suppose that $(x+y)^{m} \leq \sum_{k=0}^{m}\left(\begin{array}{c}m \\ k\end{array}\right) x^{m-k} y^{k}$, where $m \geq 1$. Then, since $y x \leq x y$ implies $y x^{m-k} y^{k} \leq x^{m-k} y^{k+1}$, it follows that

$$
\begin{aligned}
(x+y)^{m+1} & \leq(x+y) \sum_{k=0}^{m}\left(\begin{array}{c}
m \\
k
\end{array}\right) x^{m-k} y^{k} \\
& \leq \sum_{k=0}^{m}\left(\begin{array}{c}
m \\
k
\end{array}\right) x^{m+1-k} y^{k}+\sum_{k=0}^{m}\left(\begin{array}{c}
m \\
k
\end{array}\right) x^{m-k} y^{k+1}
\end{aligned}
$$




$$
\begin{aligned}
& =x^{m+1}+\sum_{k=1}^{m}\left[\left(\begin{array}{c}
m \\
k
\end{array}\right)+\left(\begin{array}{c}
m \\
k-1
\end{array}\right)\right] x^{m+1-k} y^{k}+y^{m+1} \\
& =\sum_{k=0}^{m+1}\left(\begin{array}{c}
m+1 \\
k
\end{array}\right) x^{m+1-k} y^{k} \text {. }
\end{aligned}
$$

We will also need the following results:

Theorem 3.3 ([8, Theorem 4.1(1)]). Let $(A, C)$ be an OBA with $C$ normal. If $x, y \in A$ are such that $0 \leq x \leq y$, then $\varrho(x) \leq \varrho(y)$.

We refer to the above property by saying that the spectral radius is monotone.

Theorem 3.4 ([8, Proposition 5.1]). Let $(A, C)$ be an OBA with $C$ closed and normal. If $x \in C$, then $\varrho(x) \in \operatorname{Sp}(x)$.

Theorem 3.5 ([8, Proposition 4.4]). Let $(A, C)$ be an OBA with $C$ normal. If $x, y \in C$ are such that $x y \leq y x$, then $\varrho(x y) \leq \varrho(x) \varrho(y)$ and $\varrho(y x) \leq \varrho(x) \varrho(y)$.

Proposition 3.6 ([5, Proposition 4.6)]). Let $(A, C)$ be an OBA with $C$ closed. If $x \in C$ and $\lambda>\varrho(x)$, then $(\lambda 1-x)^{-1} \geq 0$.

Finally, the following lemma follows from Theorem 3.4:

Lemma 3.7. Let $(A, C)$ be an $O B A$ with $C$ closed and normal. If $x \in C$ and $\alpha \in \mathbb{R}^{+}$, then $\varrho(x+\alpha 1)=\varrho(x)+\alpha$.

We conclude this section with an important example. Let $\mathcal{L}(X)$ denote the Banach algebra of all bounded linear operators on a Banach space $X$.

ExAMPLE 3.8. Let $E$ be a complex Banach lattice and let $C:=\{x \in E$ : $x=|x|\}$. If $K:=\{T \in \mathcal{L}(E): T C \subset C\}$, then $K$ is a closed, normal algebra cone of $\mathcal{L}(E)$. Therefore $(\mathcal{L}(E), K)$ is an $O B A$.

The nontrivial part of the above example follows from [9, Lemma 3].

4. Spectral continuity. Let $(A, C)$ be an OBA. Define, for each $x \in C$,

$$
\begin{aligned}
A(x)=\{y \in A: & x \leq y, x y \leq y x \text { or } y x \leq x y, \\
& \text { and } d(\varrho(y), \operatorname{Sp}(x)) \geq d(\alpha, \operatorname{Sp}(x)) \text { for all } \alpha \in \operatorname{Sp}(y)\} .
\end{aligned}
$$

Then $x \in A(x), A(x) \subset C$ and $A(0)=C$. In fact, it follows from Lemma 3.7 that if $C$ is closed and normal, then $A(\alpha 1)=C+\alpha 1$ for all $\alpha \in \mathbb{R}^{+}$.

It is well known that if $x$ is any element of a Banach algebra, then $\operatorname{Sp}(y) \subset$ $\operatorname{Sp}(x)+\varrho(x-y)$ for all $y$ in the commutant $\{x\}^{\mathrm{c}}$ of $x$ ([1, Theorem 3.4.1]). Theorem 4.2 shows that this inclusion continues to hold for positive elements 
$x$ of an OBA, if $y$ is an element of the set $A(x)$ rather than of $\{x\}^{\mathrm{c}}$. We need the following lemma:

Lemma 4.1. Let $A$ be a Banach algebra, $x, y \in A$ and $\alpha \in \mathbb{C}$. If $\alpha 1-x$ is invertible and $\varrho\left((\alpha 1-x)^{-1}(x-y)\right)<1$, then $\alpha 1-y$ is invertible.

Proof. If $\varrho\left((\alpha 1-x)^{-1}(x-y)\right)<1$, then $1+(\alpha 1-x)^{-1}(x-y)$ is invertible, and since $\alpha 1-y=(\alpha 1-x)\left[1+(\alpha 1-x)^{-1}(x-y)\right]$, the result follows.

THEOREM 4.2. Let $(A, C)$ be an $O B A$ with $C$ closed and normal, and let $x \in C$. Then $\operatorname{Sp}(y) \subset \operatorname{Sp}(x)+\varrho(x-y)$ for all $y \in A(x)$.

Proof. Let $y \in A(x)$. Then $0 \leq x \leq y$, so that $\varrho(x) \leq \varrho(y)$, by Theorem 3.3. If $\varrho(x)=\varrho(y)$, then $d(\varrho(y), \operatorname{Sp}(x))=0$, by Theorem 3.4, so that, by the assumption, $d(\alpha, \operatorname{Sp}(x))=0$ for all $\alpha \in \operatorname{Sp}(y)$. This implies that $d(\alpha, \operatorname{Sp}(x)) \leq \varrho(x-y)$ for all $\alpha \in \operatorname{Sp}(y)$, so that $\operatorname{Sp}(y) \subset \operatorname{Sp}(x)+\varrho(x-y)$.

So suppose that $\varrho(x)<\varrho(y)$, and suppose there exists an $\alpha \in \operatorname{Sp}(y)$ such that $d(\alpha, \operatorname{Sp}(x))>\varrho(x-y)$. By Theorem 3.4, $\varrho(y) \in \operatorname{Sp}(y)$ and hence, by the assumption, we may take $\alpha \in \mathbb{R}^{+}$with $\alpha>\varrho(x)$. Therefore

$$
\varrho\left((\alpha 1-x)^{-1}\right) \varrho(x-y)<1,
$$

with $\alpha \in \mathbb{R}^{+}$and $\alpha>\varrho(x)$. It follows from Proposition 3.6 that $(\alpha 1-x)^{-1}$ $\in C$.

If $x y \leq y x$, then $(y-x)(\alpha 1-x) \leq(\alpha 1-x)(y-x)$, so $(\alpha 1-x)^{-1}(y-x) \leq$ $(y-x)(\alpha 1-x)^{-1}$, by Lemma 3.1. It now follows from Theorem 3.5 that $\varrho\left((\alpha 1-x)^{-1}(y-x)\right) \leq \varrho\left((\alpha 1-x)^{-1}\right) \varrho(y-x)$. A similar argument yields the result in case $y x \leq x y$.

This together with 4.3 implies $\varrho\left((\alpha 1-x)^{-1}(y-x)\right)<1$. It follows from Lemma 4.1 that $\alpha \notin \mathrm{Sp}(y)$, a contradiction. Therefore $d(\alpha, \operatorname{Sp}(x)) \leq \varrho(x-y)$ for all $\alpha \in \operatorname{Sp}(y)$, so that $\operatorname{Sp}(y) \subset \operatorname{Sp}(x)+\varrho(x-y)$.

If $x$ and $y$ are commuting elements of a Banach algebra, then $\varrho(x+y) \leq$ $\varrho(x)+\varrho(y)$. In an OBA we have the following result:

Corollary 4.4. Let $(A, C)$ be an $O B A$ with $C$ closed and normal, and let $x \in C$. Then $\varrho(x+y) \leq \varrho(x)+\varrho(y)$ for all $y \in A$ such that $x+y \in A(x)$.

Proof. If $\lambda \in \operatorname{Sp}(x+y)$, then $d(\lambda, \operatorname{Sp}(x))=\left|\lambda-\mu_{\lambda}\right|$ for some $\mu_{\lambda} \in \operatorname{Sp}(x)$. It follows from Theorem 4.2 that $|\lambda| \leq\left|\lambda-\mu_{\lambda}\right|+\left|\mu_{\lambda}\right| \leq \varrho(y)+\varrho(x)$ for all $\lambda \in \operatorname{Sp}(x+y)$, so that the result follows.

Note that $x+y \in A(x)$ if and only if $y \in C, x y \leq y x$ or $y x \leq x y$ and $d(\varrho(x+y), \operatorname{Sp}(x)) \geq d(\alpha, \operatorname{Sp}(x))$ for all $\alpha \in \operatorname{Sp}(x+y)$.

Corollary 4.5. Let $(A, C)$ be an $O B A$ with $C$ closed and normal, and let $x \in C$. Then $\varrho(y) \leq \varrho(x)+\varrho(y-x)$ for all $y \in A(x)$. 
Corollary 4.6. Let $(A, C)$ be an $O B A$ with $C$ closed and normal, and let $x \in C$. Then the spectral radius is continuous at $x$, considered as an element of $A(x)$.

Proof. If $y \in A(x)$, then $\varrho(x) \leq \varrho(y)$, so that it follows from Corollary 4.5 that $|\varrho(y)-\varrho(x)| \leq \varrho(y-x) \leq\|y-x\|$.

The previous three corollaries can be strengthened. In fact, the following theorem illustrates that Corollary 4.4 continues to hold under omission of the spectral inequality in the definition of the set $A(x)$.

Theorem 4.7. Let $(A, C)$ be an $O B A$ with $C$ normal, and let $x, y \in C$ be such that either $x y \leq y x$ or $y x \leq x y$. Then $\varrho(x+y) \leq \varrho(x)+\varrho(y)$.

Proof. Let $a=\lambda^{-1} x$ and $b=\mu^{-1} y$, where $\lambda>\varrho(x)$ and $\mu>\varrho(y)$. Then $\varrho(a)<1$ and $\varrho(b)<1$. Hence, by Lemma 3.2 ,

$$
0 \leq(x+y)^{2^{n}} \leq \sum_{k=0}^{2^{n}}\left(\begin{array}{c}
2^{n} \\
k
\end{array}\right) z^{2^{n}-k} w^{k},
$$

with $z=x$ and $w=y$ or vice versa. Since $C$ is normal, there exists an $\alpha>0$ such that

$$
\left\|(x+y)^{2^{n}}\right\| \leq \alpha\left\|\sum_{k=0}^{2^{n}}\left(\begin{array}{c}
2^{n} \\
k
\end{array}\right) z^{2^{n}-k} w^{k}\right\|,
$$

and hence

$$
\left\|(x+y)^{2^{n}}\right\|^{1 / 2^{n}} \leq \alpha^{1 / 2^{n}}\left\|\sum_{k=0}^{2^{n}}\left(\begin{array}{c}
2^{n} \\
k
\end{array}\right) z^{2^{n}-k} w^{k}\right\|^{1 / 2^{n}} .
$$

It follows from Lemma 2.1(1) that

$$
\left\|(x+y)^{2^{n}}\right\|^{1 / 2^{n}} \leq \alpha^{1 / 2^{n}}(\lambda+\mu) \gamma_{n}^{1 / 2^{n}},
$$

where $\gamma_{n}=\max \left\{\left\|a^{2^{n}-k}\right\|\left\|b^{k}\right\|: 0 \leq k \leq 2^{n}\right\}$. By Lemma 2.1(2) there exists an $N \in \mathbb{N}$ such that $\gamma_{n}^{1 / 2^{n}} \leq \gamma_{N}^{1 / 2^{n}}$ for all $n \geq N$. Therefore

$$
\varrho(x+y)=\lim _{n \rightarrow \infty}\left\|(x+y)^{2^{n}}\right\|^{1 / 2^{n}} \leq \lim _{n \rightarrow \infty}\left(\alpha^{1 / 2^{n}}(\lambda+\mu) \gamma_{N}^{1 / 2^{n}}\right)=\lambda+\mu .
$$

Since this holds for every $\lambda>\varrho(x)$ and $\mu>\varrho(y)$, the result follows.

Corollary 4.8. Let $(A, C)$ be an $O B A$ with $C$ normal, and let $x, y \in C$ be such that $x \leq y$ and either $x y \leq y x$ or $y x \leq x y$. Then $\varrho(y) \leq \varrho(x)+$ $\varrho(y-x)$.

Proof. The condition $x y \leq y x$ or $y x \leq x y$ implies that $x(y-x) \leq(y-x) x$ or $(y-x) x \leq x(y-x)$. Hence $\varrho(y)=\varrho(x+(y-x)) \leq \varrho(x)+\varrho(y-x)$, since $y-x \in C$. 
Corollary 4.9. Let $(A, C)$ be an $O B A$ with $C$ normal, and let $x \in C$. Then the spectral radius is continuous at $x$, considered as an element of the set

$$
\{y \in A: x \leq y, \text { and } x y \leq y x \text { or } y x \leq x y\} .
$$

Unlike for the spectral radius function, continuity of the spectrum function Sp : $A \rightarrow K(\mathbb{C})$ (where $K(\mathbb{C})$ denotes the set of compact subsets of $\mathbb{C}$ ) does not follow from Theorem 4.2, since $x$ and $y$ cannot be interchanged in this theorem. In order to obtain continuity of the spectrum, further spectral conditions need to be imposed. This problem will not be investigated in the present note.

For each $x \in C$, consider the set

$$
D(x)=\{y \in A: x \leq y, x y \leq y x \text { or } y x \leq x y \text {, and } \delta(y) \geq \varrho(x)\} .
$$

Then $D(x) \subset C$, but $x \in D(x)$ if and only if $\operatorname{Sp}(x) \subset C(0, \varrho(x))$. Furthermore, $D(0)=C$, and if $x \in C$ is such that $C(0, \varrho(x)) \subset \operatorname{Sp}(x)$, then $D(x) \subset A(x)$. We thus have the following corollary:

Corollary 4.10. Let $(A, C)$ be an $O B A$ with $C$ closed and normal, and let $x \in C$ be such that $C(0, \varrho(x)) \subset \operatorname{Sp}(x)$. Then:

(1) $\operatorname{Sp}(y) \subset \operatorname{Sp}(x)+\varrho(x-y)$ for all $y \in D(x)$.

(2) $\varrho(x+y) \leq \varrho(x)+\varrho(y)$ for all $y \in A$ such that $x+y \in D(x)$.

(3) $\varrho(y) \leq \varrho(x)+\varrho(y-x)$ for all $y \in D(x)$.

(4) If $C(0, \varrho(x))=\operatorname{Sp}(x)$, then the spectral radius is continuous at $x$, considered as an element of $D(x)$.

However, besides being included in Corollary 4.9, Corollary 4.10(4) is also a consequence of the following property:

Proposition 4.11. Let $A$ be a Banach algebra and let $x \in A$ be such that $\operatorname{Sp}(x) \subset C(0, \varrho(x))$. Then the spectral radius is continuous at $x$.

Proof. Let $\varepsilon>0$, and $G_{\varepsilon}=\{\lambda \in \mathbb{C}: \varrho(x)-\varepsilon<|\lambda|<\varrho(x)+\varepsilon\}$. Then $\operatorname{Sp}(x) \subset G_{\varepsilon}$. If $x_{n} \rightarrow x$, then by the upper semicontinuity of the spectrum, $\operatorname{Sp}\left(x_{n}\right) \subset G_{\varepsilon}$ for all $n \geq N$, say. Since $\varrho\left(x_{n}\right)=\left|\lambda_{n}\right|$ for some $\lambda_{n} \in \operatorname{Sp}\left(x_{n}\right)$, it follows that $\varrho(x)-\varepsilon<\varrho\left(x_{n}\right)<\varrho(x)+\varepsilon$, i.e. $\left|\varrho(x)-\varrho\left(x_{n}\right)\right|<\varepsilon$ for all $n \geq N$.

To continue the discussion, define, for each $x \in C$,

$$
\begin{aligned}
& B(x)=\{y \in A: x \leq y, x y \leq y x \text { or } y x \leq x y \\
&\text { and } \left.(\alpha 1-x)^{-1} \in C \text { for all } \alpha \in \operatorname{Sp}(y) \backslash \operatorname{Sp}(x)\right\} .
\end{aligned}
$$

Then $x \in B(x), B(x) \subset C$ and $B(0)=\{y \in C: \alpha 1 \in C$ for all $\alpha \in \operatorname{Sp}(y)\}$ if $C$ is inverse-closed. As in the case of $A(x)$ we have the following theorem:

TheOREM 4.12. Let $(A, C)$ be an $O B A$ with $C$ normal, and let $x \in C$. Then $\operatorname{Sp}(y) \subset \operatorname{Sp}(x)+\varrho(x-y)$ for all $y \in B(x)$. 
Proof. Let $y \in B(x)$. Suppose there exists an $\alpha \in \operatorname{Sp}(y)$ such that $d(\alpha, \operatorname{Sp}(x))>\varrho(x-y)$. Then

$$
\varrho\left((\alpha 1-x)^{-1}\right) \varrho(x-y)<1 .
$$

If $x y \leq y x$, then $(y-x)(\alpha 1-x) \leq(\alpha 1-x)(y-x)$, so that $(\alpha 1-x)^{-1}(y-x) \leq$ $(y-x)(\alpha 1-x)^{-1}$, by Lemma 3.1. Since $y \in B(x)$, we have $y-x \in C$ and $(\alpha 1-x)^{-1} \in C$, so Theorem 3.5 shows that $\varrho\left((\alpha 1-x)^{-1}(y-x)\right) \leq$ $\varrho\left((\alpha 1-x)^{-1}\right) \varrho(y-x)$. A similar argument yields the result in case $y x \leq x y$. This together with (4.13) implies $\varrho\left((\alpha 1-x)^{-1}(y-x)\right)<1$. By Lemma 4.1, $\alpha \notin \operatorname{Sp}(y)$, which is a contradiction, and hence the result follows.

Finally, for each $x \in C$ set

$$
\begin{aligned}
E(x)=\{y \in A: x \leq y, x y \leq y x \text { or } y x \leq x y, & \\
& \text { and } x \leq \alpha 1 \text { for all } \alpha \in \operatorname{Sp}(y) \backslash \operatorname{Sp}(x)\} .
\end{aligned}
$$

Then $x \in E(x)$ and $E(x) \subset C$. Furthermore, $E(0)=\{y \in C: \alpha 1 \in C$ for all $\alpha \in \operatorname{Sp}(y)\}$, and if $C$ is inverse-closed, then $E(x)=B(x)$.

In conclusion, we have the following corollary:

Corollary 4.14. Let $(A, C)$ be an $O B A$ with $C$ normal, and let $x \in C$. If either $C$ is inverse-closed or $E(x) \subset B(x)$, then $\operatorname{Sp}(y) \subset \operatorname{Sp}(x)+\varrho(x-y)$ for all $y \in E(x)$.

5. Examples. As mentioned before, the results in Section 4 are known to hold for elements commuting with $x$. Therefore, to show that these results are indeed applicable, we supply some examples showing that the sets defined in Section 4 contain elements which do not commute with $x$.

EXAMPLE 5.1. Let $A$ be the set of upper triangular $2 \times 2$ complex matrices, $l^{\infty}(A)$ the set

$$
\left\{x=\left(x_{1}, x_{2}, \ldots\right): x_{i} \in A \text { for all } i \in \mathbb{N} \text { and }\left\|x_{i}\right\|_{A} \leq K_{x} \text { for all } i \in \mathbb{N}\right\},
$$

and $C$ the set

$$
\left\{\left(c_{1}, c_{2}, \ldots\right) \in l^{\infty}(A): c_{i} \text { has only nonnegative entries for all } i \in \mathbb{N}\right\} .
$$

Then $\left(l^{\infty}(A), C\right)$ is an (infinite-dimensional) $O B A, C$ is closed and normal, and for at least some $x \in C$ the sets $E(x), B(x)$ and $A(x)$ contain elements which do not commute with $x$.

Proof. A proof of the fact that $\left(l^{\infty}(A), C\right)$ is an OBA with $C$ normal was given in [4, Example 4.16]. Closedness of $C$ follows easily from the definition of $C$. Let

$$
x=\left(\left(\begin{array}{ll}
1 & 0 \\
0 & 0
\end{array}\right),\left(\begin{array}{ll}
1 & 0 \\
0 & 0
\end{array}\right), \ldots\right) .
$$


Then $x \in C$ and $\operatorname{Sp}(x)=\{0,1\}$. Let

$$
y=\left(\left(\begin{array}{ll}
1 & 1 \\
0 & 2
\end{array}\right),\left(\begin{array}{ll}
1 & 1 \\
0 & 2
\end{array}\right), \ldots\right) .
$$

Then $x \leq y$ and $\operatorname{Sp}(y)=\{1,2\}$. Since

$$
x y=\left(\left(\begin{array}{ll}
1 & 1 \\
0 & 0
\end{array}\right),\left(\begin{array}{ll}
1 & 1 \\
0 & 0
\end{array}\right), \ldots\right), \quad y x=\left(\left(\begin{array}{ll}
1 & 0 \\
0 & 0
\end{array}\right),\left(\begin{array}{ll}
1 & 0 \\
0 & 0
\end{array}\right), \ldots\right),
$$

we have $y x \leq x y$. The only element of $\operatorname{Sp}(y) \backslash \operatorname{Sp}(x)$ is 2 , and

$$
(2 \cdot 1-x)^{-1}=\left(\left(\begin{array}{cc}
1 & 0 \\
0 & 1 / 2
\end{array}\right),\left(\begin{array}{cc}
1 & 0 \\
0 & 1 / 2
\end{array}\right), \ldots\right) \in C .
$$

Therefore $y \in B(x)$. In fact, $x \leq 2 \cdot 1$, so that $y \in E(x)$. Moreover, although $C$ is not inverse-closed, $E(x)=B(x)$.

Since $d(\varrho(y), \operatorname{Sp}(x))=1$ and $\{d(\alpha, \operatorname{Sp}(x)): \alpha \in \operatorname{Sp}(y)\}=\{0,1\}$, it follows that $y \in A(x)$ as well. Furthermore, it is easily checked that $x+y \in$ $A(x) \cap E(x) \subset B(x)$.

Consequently, the following results apply to $\left(l^{\infty}(A), C\right)$ : Theorem 4.2 , Corollaries 4.4, 4.5 and 4.6, Theorem 4.7, Corollaries 4.8 and 4.9, Theorem 4.12 and Corollary 4.14. (Alternatively, for $l^{\infty}(A)$, Corollaries 4.6 and 4.9 follow directly from the fact that the spectrum of every element of $l^{\infty}(A)$ is totally disconnected. This is Newburgh's Theorem [1, Corollary 3.4.5].)

EXAMPLE 5.2. Consider, for any $p$ with $1 \leq p \leq \infty$, the complex Banach lattice $l^{p}$, and let $A=\mathcal{L}\left(l^{p}\right), C=\left\{x \in l^{p}: x=|x|\right\}$ and $K=\left\{T \in \mathcal{L}\left(l^{p}\right)\right.$ : $T C \subset C\}$. Then $(A, K)$ is an $O B A$ with $K$ closed and normal, and for some $S \in K$ such that $\operatorname{Sp}(S)=C(0, \varrho(S))$, the sets $D(S)$ and $A(S)$ contain elements which do not commute with $S$.

Proof. The first statement follows from Example 3.8. Let $S\left(\xi_{1}, \xi_{2}, \ldots\right)$ $=\left(0, \xi_{1} / 1, \xi_{2} / 2, \ldots\right)$. Then $S \in K$ and $\operatorname{Sp}(S)=\{0\}=C(0, \varrho(S))$. Let $T\left(\xi_{1}, \xi_{2}, \ldots\right)=\left(0, \xi_{1}, \xi_{2}, \ldots\right)$. Then $S \leq T$ and $\operatorname{Sp}(T)=\bar{D}(0,1)$, so that $\delta(T)=0=\varrho(S)$. Since we have $(S T)\left(\xi_{1}, \xi_{2}, \ldots\right)=\left(0,0, \xi_{1} / 2, \xi_{2} / 3, \ldots\right)$ and $(T S)\left(\xi_{1}, \xi_{2}, \ldots\right)=\left(0,0, \xi_{1} / 1, \xi_{2} / 2, \ldots\right)$, it follows that $S T \leq T S$, and therefore $T \in D(S)$. In addition, clearly $S+T \in D(S)$. (Hence, also $T \in A(S)$ and $S+T \in A(S)$.)

In the case of $(A, K)$ of the above example, the applicable results are: Theorem 4.2, Corollaries 4.4, 4.5 and 4.6, Theorem 4.7 and Corollaries 4.8, 4.9 and 4.10 .

Acknowledgements. The author thanks one of the referees for suggesting the existence of the stronger versions (Theorem 4.7 and Corollaries 4.8 and 4.9) of Corollaries 4.4, 4.5 and 4.6. 


\section{References}

[1] B. Aupetit, A Primer on Spectral Theory, Springer, New York, 1991.

[2] L. Burlando, Continuity of spectrum and spectral radius in Banach algebras, in: Functional Analysis and Operator Theory, J. Zemánek (ed.), Banach Center Publ. 30, Inst. Math., Polish Acad. Sci., Warszawa, 1994, 53-100.

[3] A. R. Esajan [A. R. Esayan], On estimating the spectrum of the sum of positive semi-commuting operators, Sibirsk. Mat. Zh. 7 (1966), 460-464 (in Russian); English transl.: Siberian Math. J. 7 (1966), 374-378.

[4] H. du T. Mouton and S. Mouton, Domination properties in ordered Banach algebras, Studia Math. 149 (2002), 63-73.

[5] S. Mouton, A spectral problem in ordered Banach algebras, Bull. Austral. Math. Soc. 67 (2003), 131-144.

[6] -, Convergence properties of positive elements in Banach algebras, Math. Proc. R. Ir. Acad. Sect. A 102 (2002), 149-162.

[7] S. Mouton (née Rode) and H. Raubenheimer, More spectral theory in ordered Banach algebras, Positivity 1 (1997), 305-317.

[8] H. Raubenheimer and S. Rode, Cones in Banach algebras, Indag. Math. (N.S.) 7 (1996), 489-502.

[9] H. H. Schaefer, Some spectral properties of positive linear operators, Pacific J. Math. 10 (1960), 1009-1019.

[10] V. Ja. Stecenko [V. Ya. Stetsenko], On a spectral property of an indecomposable operator, Uspekhi Mat. Nauk 22 (1967), no. 3, 242-244 (in Russian).

[11] M. Zima, On the local spectral radius in partially ordered Banach spaces, Czechoslovak Math. J. 49 (124) (1999), 835-841.

Department of Mathematics

University of Stellenbosch

Private Bag X1

Matieland 7602, South Africa

E-mail: smo@sun.ac.za

Received December 14, 2004

Revised version January 31, 2006 